\title{
Characterizing major agricultural land change trends in the Western Corn Belt
}

\author{
Yang Shao $^{\mathrm{a}, *}$, Gregory N. Taff ${ }^{\mathrm{b}}$, Jie Ren ${ }^{\mathrm{a}}$, James B. Campbell ${ }^{\mathrm{a}}$ \\ ${ }^{a}$ Virginia Tech, College of Natural Resources and Environment, Geography Department, 115 Major Williams Hall, Blacksburg, VA 24061, USA \\ ${ }^{\mathrm{b}}$ Norwegian Forest and Landscape Institute - Northern Office, Tromsø 9037, Norway
}

\section{A R T I C L E I N F O}

\section{Article history:}

Received 3 May 2016

Received in revised form 11 October 2016

Accepted 24 October 2016

Available online 11 November 2016

\section{Keywords:}

Cropland mapping

Neural network

Threshold-moving

MODIS

\begin{abstract}
A B S T R A C T
In this study we developed annual corn/soybean maps for the Western Corn Belt within the United States using multi-temporal MODIS NDVI products from 2001 to 2015 to support long-term cropland change analysis. Based on the availability of training data (cropland data layer from the USDA-NASS), we designed a cross-validation scheme for 2006-2015 MODIS data to examine the spatial generalization capability of a neural network classifier. Training data points were derived from a three-state subregion consisting of North Dakota, Nebraska, and Iowa. Trained neural networks were applied to the testing sub-region (South Dakota, Kansas, Minnesota, and Missouri) to generate corn/soybean maps. Using a default threshold value (neural network output signal $\geqslant 0.5$ ), the neural networks performed well for South Dakota and Minnesota. Overall accuracy was higher than 80\% (kappa $>0.55$ ) for all testing years from 2006 to 2015. However, we observed high variation of classification performance for Kansas (overall accuracy: 0.71-0.82) and Missouri (overall accuracy: 0.65-0.77) for various testing years. We developed a threshold-moving method that decreases/increases threshold values of neural network output signals to match MODIS-derived corn/soybean acreage with the NASS acreage statistics. Over 70\% of testing states and years showed improved classification performance compared to the use of a default 0.5 threshold. The largest improvement of kappa value was about 0.08 . This threshold-moving method was used to generate MODIS-based annual corn/soybean map products for 2001-2015. A non-parametric Mann-Kendall test was then used to identify areas that showed significant $(p<0.05)$ upward/downward trends. Areas showing fast increase of corn/soybean intensities were mainly located in North Dakota, South Dakota, and the west portion of Minnesota. The highest annual increase rate for a 5-km moving window was about $6.8 \%$.
\end{abstract}

(C) 2016 International Society for Photogrammetry and Remote Sensing, Inc. (ISPRS). Published by Elsevier B.V. All rights reserved.

\section{Introduction}

From the early 2000s until now, the US Corn Belt has experienced a significant land use and land cover change (LUCC) event characterized by conversion of grasslands and wetlands to croplands (Wright and Wimberly, 2013) and intensive corn/soybean production (Lunetta et al., 2010; Sahajpal et al., 2014; Ren et al., 2016). Assessing LUCC in such a large agricultural landscape presents an important challenge, given that agricultural land use is continuously changing and may have sharp year-to-year differences. The Cropland Data Layer (CDL), developed by the United States Department of Agriculture - National Agricultural Statistics Service (USDA-NASS), is currently the only ready-to-use data

\footnotetext{
* Corresponding author.

E-mail address: yshao@vt.edu (Y. Shao).
}

product for evaluating large-scale agricultural land change at annual intervals (Boryan et al., 2011; Hansen and Loveland, 2012; Lark et al., 2015). Using CDLs, researchers are just beginning to characterize and understand rates and patterns of agriculturalspecific LUCC in the Corn Belt. For example, Wright and Wimberly (2013) examined CDLs from 2006 to 2011 and reported a 1.0-5.4\% of annual grass-to-corn/soy conversion in the Western Corn Belt (WCB). Their research mainly focused on two temporal snapshots (2006 and 2011), thus the authors suggested that change rates may be attributed to both an underlying trend of land use change and short-term crop rotation patterns (e.g., grassland/ hay-corn/soybean rotation). Analyses of longer-term annual corn/soybean map products may reduce the confusion from short-term crop rotation patterns and highlight overall change trends and spatial patterns. 
Complete CDLs are now available for the conterminous United States for 2008-2015. The spatial resolution of CDLs ranges from $30 \mathrm{~m}$ to $56 \mathrm{~m}$, depending on the satellite data source used and processing protocols. Older CDLs, however, are not available for several states in the WCB region. For Kansas, Minnesota, Missouri, and South Dakota, CDLs are entirely missing for 2001-2005 (Fig. 1). Without CDLs for 2001-2005 as baseline datasets, it is a challenge to conduct a thorough analysis of corn/soybean-related agricultural land changes and examine their links to key US biofuel energy policies such as the Energy Policy Act of 2005 and the Energy Independence and Security Act of 2007. Developing CDLs requires multiple cloud-free images for each crop-growing season. It also requires good training/validation data. In the early 2000s, the USDA-NASS primarily used NASS June Area Survey (JAS) data as training/validation and an in-house software package (Peditor) for developing CDLs. In 2006-2007, NASS started to use high quality training/validation data points derived from detailed Common Land Unit (CLU) data from the Farm Service Agency (FSA) and commercial software (See5 Decision Tree) to improve CDL quality (Johnson, 2010; Johnson and Mueller, 2010; Boryan et al., 2011). By 2008, CDLs are considered nationally operational and the production was expanded to include all 48 conterminous states in the US. The availability of high quality training data (e.g., CLU from FSA) is one of the main reasons that the USDA-NASS did not provide high quality CDLs for the conterminous US before 2008. It is unclear whether the USDA-NASS plans to generate CDLs backward in time to extend the available time-series of cropland map products.

Several studies showed promise for multi-temporal Moderate Resolution Imaging Spectroradiometer (MODIS) Normalized Difference Vegetation Index (NDVI) data to perform crop-specific mapping with reasonable accuracy (Chang et al., 2007; Doraiswamy et al., 2007; Wardlow and Egbert, 2008; Shao et al., 2010; Wardlow and Egbert, 2010; Zhang et al., 2014; Chen et al., 2016; Zhong et al., 2016). However, many of these studies focused on short-term (e.g., 1-3 year) image classification experiments and their methods have yet to be expanded for annual cropland mapping. Furthermore, it was found that the main summer crops such as corn and soybean have similar spectral-temporal signals that typically lead to moderate-low cropland map accuracy (Wardlow et al., 2007; Shao et al., 2010). A combined corn/soybean class, as defined by Wright and Wimberly (2013), may substantially improve thematic map accuracy to support longer-term cropland change analysis. Both corn and soybean are considered to be intensive cropping compared to hay/pasture or natural lands. Analyzing trends in corn/soybean planting thus might be sufficient to characterize key agricultural intensification processes and patterns in the WCB.

Annual corn/soybean mapping with multi-temporal MODIS data requires high quality training data points for image classification. The currently available, but spatially-limited, 2001-2005 CDLs (North Dakota, Nebraska and Iowa, Fig. 1) could be used for reference to generate a large number of training data points for corn/soybean mapping. This possibility raises an interesting question of the capability of spatial generalization of US crop mapping through image classification - whether a trained multi-temporal classifier for a region can be directly applied to a different region (Kansas, Minnesota, Missouri, and South Dakota) where CDLs are not available. There is no guarantee that a trained classifier would produce acceptable results because corn/soybean phenologies and general land cover compositions may vary substantially across regions. Previous published studies showed promising results for Neural Networks (NN) and Random Forest in both spatial and temporal generalization (Shao and Lunetta, 2012; H. Wang et al., 2014,
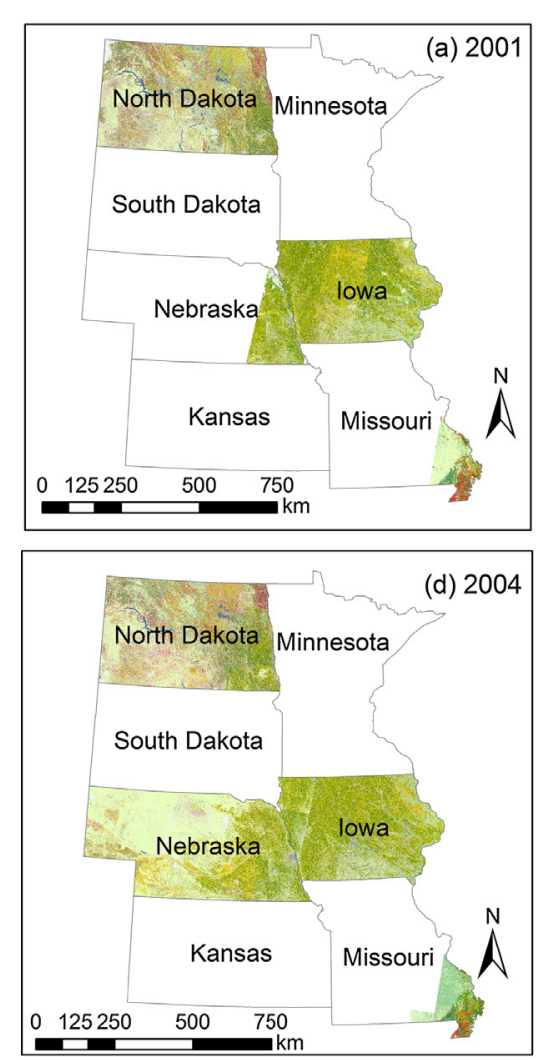
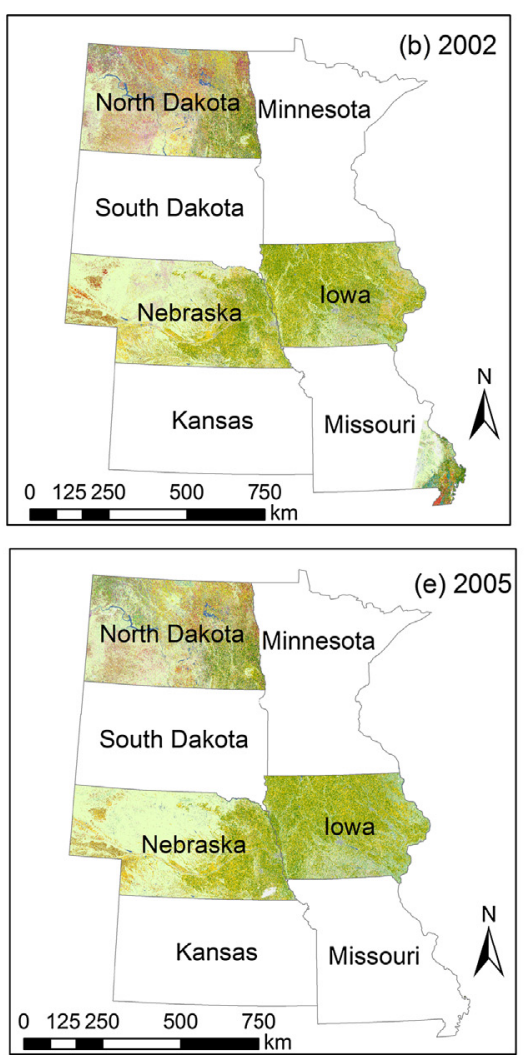
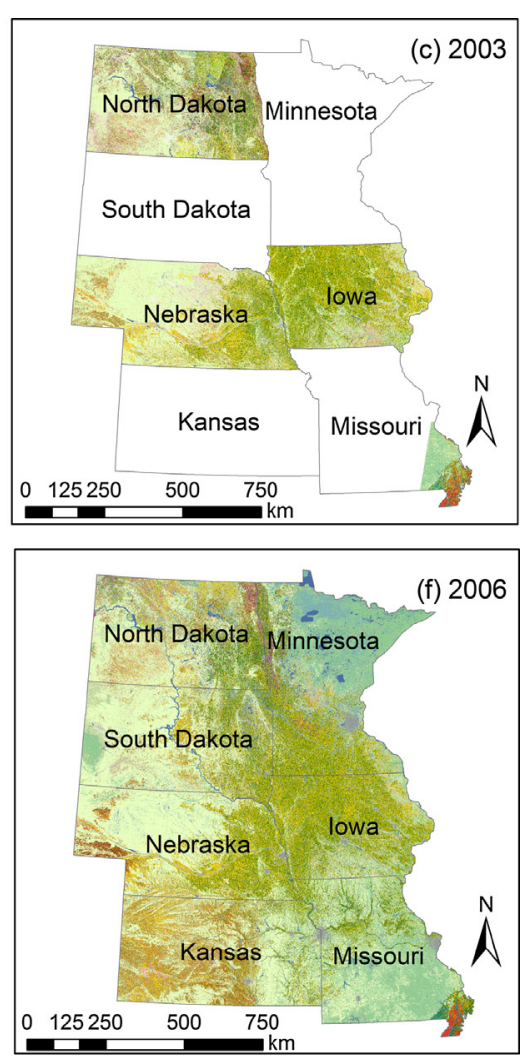

Fig. 1. Spatial coverage of the Cropland Data Layer for the Western Corn Belt, 2001-2006. 
Q. Wang et al., 2014). There is a need to examine their spatial generalization capability for long-term operational corn/soybean mapping tasks.

Another useful resource for corn/soybean mapping is the annual NASS crop acreage statistics (e.g., corn/soybean planted) at state or county levels. The NASS acreage statistics are mainly derived through survey-statistical methods and routinely provided at national, state, and county levels. These numbers are often different from results of remote sensing pixel counting (e.g., CDLs) at aggregated levels. The NASS acreage statistics have been commonly used for accuracy assessment of remote sensing cropland mapping (Chang et al., 2007; Wardlow and Egbert, 2008). Good agreement between remote sensing-derived corn/soybean acreage and the NASS acreage statistics generally suggests acceptable cropland mapping results. Few previously published studies have effectively integrated the NASS statistics for improving remote sensing cropland mapping, other than for use in accuracy assessment.

Many classification algorithms can be trained to generate fuzzy outputs or approximate posterior probabilities (Richard and Lippmann, 1991; Moody et al., 1996; Shao et al., 2011). An important practice within the machine learning community is that classification performance can be significantly improved by simply adjusting the output threshold value instead of using a default one (e.g., 0.5) to label each target class (Provost, 2000; Zhou and Liu, 2006). This threshold-moving method has not been routinely applied in land cover mapping application, especially for spatial generalization problems where differences in spectral/temporal signals across regions may require adjustments in classification methods when compared to applications where training data are derived from the region where the classification is performed.

The main objective of this study was to develop annual MODISbased corn/soybean map products for 2001-2015 to support longterm agricultural land change analysis in the WCB states. We use a NN classifier to address the following specific research objectives: (1) examine whether a classifier trained for one region can be spatially generalized to another region; spatial generalization performance was assessed for study years of 2006-2015 since reference CDLs are available for the entire WCB study region; (2) develop a classifier output threshold-moving method by integrating the NASS statistics; our main motivation was to use the NASS acreage statistics to support automated threshold value selection for the NN classification; and (3) generate annual corn/soybean map products and characterize long-term corn/soybean change trends for the period 2001-2015.

\section{Methods}

\subsection{Study area and data preparation}

The study area for MODIS-based annual corn/soybean mapping was the WCB region include seven main corn/soybean producing states: North Dakota, South Dakota, Nebraska, Kansas, Minnesota, Iowa, and Missouri. We obtained 2001-2015 Terra-MODIS $250 \mathrm{~m}$ 16-day composite of vegetation index data (MOD13Q1, Collection 5) from the NASA Reverb website (http://reverb.echo.nasa.gov/) for the WCB. MODIS NDVI mosaics were created for each of the 16-day composite images and then projected to an Albers EqualArea Conic projection. The 16-day MODIS NDVI data were stacked to build a time-series data cube. MODIS pixels with quality issues were identified using the reliability index (RI) and new values were estimated for these pixels through a Whittaker smoother (Eilers, 2003; Atzberger and Eilers, 2011; Shao et al., 2016). The Whittaker smoother was implemented in the Matlab environment and the smoothing parameter was automatically adjusted through a pixel-by-pixel cross-validation approach (Eilers, 2003). The cleaned NDVI time-series data were then clipped to the 7-State WCB boundary.

All available CDLs from 2001 to 2015 for the WCB were downloaded from the USDA-NASS CropScape (http://nassgeodata.gmu. edu/CropScape/). For major crop types such as corn and soybean, the USDA-NASS reported high classification accuracies of over $85 \%$ for all states. We expected that the combined corn/soybean class has even higher accuracy, because the confusion between these two classes is reduced. Using annual CDLs as input, we calculated proportional corn/soybean area within each $250 \mathrm{~m}$ MODIS grid. For each year from 2001 to 2015 , we then generated a binary corn/soybean (1) and other class (0) map using 50\% proportional corn/soybean as a threshold. These corn/soybean binary map products were used as the primary reference data to support MODISbased annual corn/soybean mapping. We stacked the 2006-2015 corn/soybean binary maps to build a large corn/soybean mask layer, which includes all pixels used for at least one year out of ten total years. This large corn/soybean mask was used to remove the pixels that were never corn/soybean. Our assumption is that corn/soybean fields have been expanding since 2001, therefore a mask from later years (2006-2015) would include all corn/soybean fields in 2001-2005. We verified this for states of North Dakota and Iowa, where CDLs are available for 2001-2005; the large corn/soybean mask covered above $99 \%$ of corn/soybean pixels for each individual year from 2001 to 2005. Our annual corn/soybean mapping efforts were conducted within this large cropland mask layer. A similar approach has been used in Johnson's (2013) annually tilled cropland mapping and Shao et al.'s (2015) crop yield prediction work. We also obtained the USDA-NASS state level agricultural statistics from http://www.nass.usda.gov/Quick_Stats/. Corn and soybean planted acreage statistics were obtained for the WCB states for 2001-2015.

\subsection{Corn/soybean mapping through NN spatial generalization}

CDLs from North Dakota, Nebraska and Iowa were used as reference to select training data points for MODIS-NDVI classification. For each study year from 2006 to 2015, a number of training datasets were examined by randomly selecting $1 / 2000,1 / 1500,1 / 1000$, $1 / 500,1 / 100$, and $1 / 20$ of total MODIS pixels to evaluate the impacts of training sample size on classification results. We used a three-layer perceptron NN to separate corn/soybean from the other class. The output layer consisted of one output node (corn/soybean $=1$; other $=0$ ) for this two-class classification problem. The NN input layer included 13 nodes representing thirteen 16day MODIS NDVI layers from Julian day-of-year (DOY) 97 to 289. This subset of multi-temporal MODIS NDVI data was used since winter-early spring images for the WCB region have significant snow cover, and do not provide valuable information in corn/soybean mapping (Shao et al., 2011). Different numbers of nodes (20, 30 , and 40 ) in the NN hidden layer were tested. Additional adjustable NN parameters included learning rate and momentum. To achieve high generalization performance, each training dataset was further divided into 5 sets and a 5 -fold cross-validation was used to determine the optimal NN parameters.

For each year from 2006 to 2015, the trained NN networks were applied to multi-temporal MODIS-NDVI data for the other states, Kansas, Minnesota, Missouri, and South Dakota. The NN outputs were retained in fuzzy values from 0 to 1 , which allowed us to adjust threshold values to generate a range of corn/soybean map products. We first used a default threshold value (0.5) to develop corn/soybean maps. The 2006-2015 CDLs for the abovementioned four states were used to assess map accuracy. Overall accuracy, kappa coefficient, and user's and producer's accuracies were calculated (Congalton, 1991). To further evaluate the NN model's spatial generalization performances, we designed a simple 
control to benchmark map accuracy for the four-state testing region. Specifically, we randomly selected training data points e.g., (1/2000 to $1 / 20$ of total points) from this four-state region. Independent NN training and testing were then conducted to classify this same four-state region to generate the benchmark map accuracies. Classification accuracies from the spatial generalization and benchmark approach were compared for the entire four-state region as well as each individual state. We were particularly interested in differences in classification accuracies between the spatial generalization and the benchmark approaches. Similar accuracy levels would indicate a potential solution in generating corn/soybean map products for 2001-2005 using a spatial generalization of NN classification.

\subsection{Integration of NN threshold-moving and the NASS statistics}

From the spatial generalization approach, the NN output signals were retained in a fuzzy manner (i.e., between 0 and 1 ) and we examined how different threshold values affect crop/soybean mapping performance. For each study year from 2006 to 2015, we examined all potential threshold values from 0.2 to 0.8 with 0.02 incremental steps. At each step, the initial fuzzy corn/soybean map was converted to a binary value ( $\operatorname{corn} /$ soybean $=1$, others $=0$ ). Each binary corn/soybean map was compared to the $250 \mathrm{~m}$ corn/soybean binary map derived from the CDLs. Overall accuracies and kappa statistics were computed for different threshold values.

For each threshold value, the total number of corn/soybean pixels from the MODIS-derived binary map was summarized to estimate the remote sensing-based corn/soybean acreage. Such remote sensing-based crop/soybean acreages were compared to the NASS statistics to support automated threshold selection. An 'optimal' threshold could be identified where remote sensingderived corn/soybean acreage is in agreement with the NASS acreage statistics. It should be noted that the NASS has reported that such remote sensing pixel counting approaches could be downwardly biased compared to the official NASS acreage statistics (Gallego, 2004). The NASS acreage statistics are obtained through standard survey-statistical methods (Boryan et al., 2011). To examine the general relationship between the remote sensing-derived acreage and the NASS statistics, we computed the simple ratio between the CDL-derived corn/soybean acreage and the NASS statistics for each state and then obtained an average ratio for 2010-2015:

$R=\frac{1}{n} \sum_{1}^{n}\left(\frac{\text { CDL_area }_{i}}{\text { NASS_area }_{i}}\right)$

where CDL_area denotes the corn/soybean acreage estimated through CDL pixel counting and NASS_area is the official NASS corn/soybean acreage. The main reason to focus on 2010-2015 was that NASS continued developing their CDL processing protocols and the CDL products in recent years tend to have higher consistency (Kline et al., 2013). We also note that the calculation of total acreage from the binary corn/soybean map is based on previously processed CDLs to have a proportional corn/soybean value in each $250 \mathrm{~m}$ pixel, with a 0.5 threshold value used to generate binary corn/soybean map. The rescaling of CDLs was designed to match the $250 \mathrm{~m}$ MODIS spatial resolution.

We hoped that there would be a relatively stable ratio between CDL-derived acreage and the NASS acreage statistics across different study years for each state. The average ratio (R) for each state can then combined with the annual state-level NASS statistics to estimate total corn/soybean acreage (pixel counts at $250 \mathrm{~m}$ scale) for any given study year. With such MODIS pixel counts, we could identify an 'optimal' threshold value by applying the threshold-moving approach (0.2-0.8 with 0.02 incremental steps) to the NN output signals. It should be noted that the 'optimal' threshold is associated with NASS statistics and it may not always be linked to optimal classification performance at pixel-by-pixel scale. This automated threshold selection method was repeated for each study year and state to produce the NASS-adjusted corn/soybean map products. We then evaluated the per-pixel classification accuracies of the NASS-adjusted corn/soybean map products using overall accuracy and kappa statistics.

\subsection{Analysis of long-term trends of corn/soybean planting from 2001 to 2015}

Classification accuracies from (1) the simple spatial generalization and (2) the threshold-moving approaches were compared using the 2006-2015 experimental data. The classification results with highest overall accuracy (and kappa coefficient) were used to support the long-term land change analysis. Percent corn/soybean area, or "crop intensity" values, were calculated for $5 \mathrm{~km}$ by $5 \mathrm{~km}$ moving windows (Lunetta et al., 2010). The long-term (2001-2015) crop intensity trends were then examined windowby-window using a non-parametric Mann-Kendall test. The Mann-Kendall test evaluates the monotonic downward/upward trend over time. One appealing feature of the Mann-Kendall test is its robustness to outliers (Hamed, 2008). For each $5 \mathrm{~km}$ window showing significant upward/downward trend, we used Sen's slope estimator to assess the average annual change rate. Sen's slope is a nonparametric alternative for estimating a slope (change rate) and it is not sensitive to outliers.

\section{Results}

\subsection{NN spatial generalization without threshold moving}

Fig. 2(a) and (b) compare overall classification accuracies from the spatial generalization approach and the benchmark approach for the testing region. Each box presents summary statistics of overall accuracies (or kappa coefficients) from 2006 to 2015. Using a spatial generalization approach and a default threshold value of 0.5 , the average overall accuracy ranged from 0.77 to 0.79 when training data points increased from 0.05 percent to 5 percent of the total MODIS pixels. The average overall accuracy for the benchmark approach was 0.80 and 0.82 using 0.05 percent and 5 percent of total MODIS pixels, respectively. On average, there was about 3\% difference of overall accuracy. The variability of the overall accuracy (and kappa value) was slightly smaller when more training data points were used for NN training. In general, classification performance tended to saturate when $1 \%$ of total MODIS pixels were used for training and there was no significant improvement when more training data points were added to the NN training.

The classification performance was further examined for each state within the four-state testing-region (Table 1). We focused on results derived from the largest training datasets (5\% of total MODIS pixels) where the highest overall classification accuracies were obtained. For Minnesota and South Dakota, the lowest classification accuracy in one year was 0.82 and 0.80 , respectively, suggesting good overall performance of spatial generalization approach. More importantly, the classification accuracy statistics from the spatial generalization and benchmark approach were quite similar for the mean, minimum, and maximum values. For Kansas and Missouri, the average overall accuracy for 2006-2015 was 0.77 (kappa $=0.49$ ) and 0.73 (kappa $=0.46)$. Such accuracy statistics are acceptable for most remote sensing land cover mapping studies, however the overall accuracies were about $4 \%$ and $5 \%$ lower than the benchmark statistics. Furthermore, the lowest 

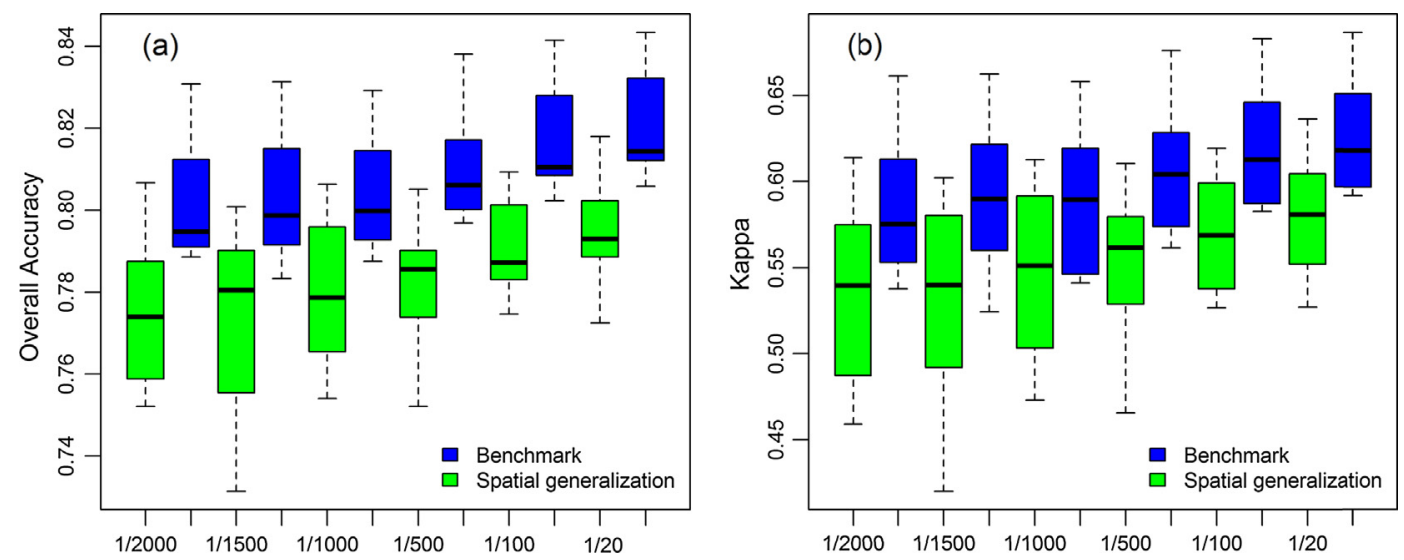

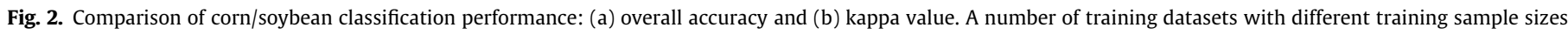
were examined using spatial generalization and benchmark approaches.

Table 1

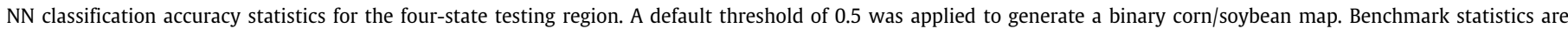
provided as if training data were available for the four-state region.

\begin{tabular}{|c|c|c|c|c|c|c|}
\hline \multirow[t]{2}{*}{ Kansas } & \multicolumn{2}{|c|}{ Mean (2006-2015) } & \multicolumn{2}{|c|}{ Minimum (2006-2015) } & \multicolumn{2}{|c|}{ Maximum (2006-2015) } \\
\hline & SG & Benchmark & SG & Benchmark & SG & Benchmark \\
\hline Overall & 0.77 & 0.81 & 0.71 & 0.78 & 0.82 & 0.85 \\
\hline Kappa & 0.49 & 0.59 & 0.40 & 0.56 & 0.56 & 0.64 \\
\hline \multicolumn{7}{|l|}{ Minnesota } \\
\hline Overall & 0.84 & 0.85 & 0.82 & 0.83 & 0.86 & 0.86 \\
\hline Kappa & 0.61 & 0.63 & 0.56 & 0.57 & 0.69 & 0.69 \\
\hline \multicolumn{7}{|l|}{ Missouri } \\
\hline Overall & 0.73 & 0.78 & 0.65 & 0.76 & 0.77 & 0.81 \\
\hline Kappa & 0.46 & 0.55 & 0.33 & 0.50 & 0.53 & 0.61 \\
\hline \multicolumn{7}{|c|}{ South Dakota } \\
\hline Overall & 0.82 & 0.83 & 0.80 & 0.82 & 0.84 & 0.85 \\
\hline Kappa & 0.61 & 0.64 & 0.55 & 0.57 & 0.67 & 0.70 \\
\hline
\end{tabular}

classification accuracy for a given year was 0.71 and 0.65 for Kansas and Missouri; both were substantially lower compared to the benchmark statistics (0.78 and 0.76). Kansas is a major winter wheat-producing state, recognized for double-cropping (winter wheat followed by a late planted soybean) in some areas of the state. This effect leads to much of the soybean crop in Kansas having a different phenology from soybeans in other states, which likely contributed to reduced accuracy here. Such differences in cropping practices or phenology thus potentially limit NN spatial generalization effectiveness. In addition, Kansas and Missouri are located beyond the southern border of the extent of the training data, which may lead to phenologies that are not so well-trained for within the training data. The states with the best accuracy, Minnesota and South Dakota, may exhibit such accuracies because they are located mostly within the geographic extent of regions where training data were selected. In addition, their dominant corn/soybean summer crop may contribute to relatively higher classification performance.

\subsection{Impacts of $N N$ threshold-moving on classification performance}

Classification performance was highly dependent on the threshold selection to label the corn/soybean pixels. We use image classification results for Missouri as an example to illustrate the impacts of threshold-moving. Fig. 3(a) and (b) shows variations of overall accuracies and kappa coefficients by moving threshold values from 0.2 to 0.8 for 2012 corn/soybean classification for Missouri. The overall accuracies ranged from 0.51 to 0.70 (kappa 0.150.37). A default 0.5 threshold value led to an overall accuracy of 0.65 (kappa $=0.33$ ). The optimal threshold value should be around 0.3 , a much smaller threshold value compared to a default 0.5 . Fig. 3(c) shows the ratio of MODIS-derived corn/soybean acreage to the NASS acreage statistics. For 2012, the NASS-reported official corn/soybean acreage was about $36,422 \mathrm{~km}^{2}$ in Missouri. Depending on the various threshold values $(0.2-0.8)$ selected, MODISestimated acreage ranged from 36,427 to $8741 \mathrm{~km}^{2}$, corresponding to MODIS_area/NASS_area ratio of $1.00-0.24$. Such results were expected because fewer MODIS pixels were labeled as corn/soybean when the threshold increased to higher values. The highest image classification accuracy was associated with a ratio value of around 0.83 .

Table 2 compares classification confusion matrices and accuracy statistics for three selected threshold values of $0.3,0.5$ and 0.7 . The threshold value of 0.3 generated the highest overall accuracy of $70.2 \%$ (kappa $=0.37$ ). The user's and producer's accuracy for corn/soybean class was $77.1 \%$ and $75.0 \%$, respectively. For a default threshold value of 0.5 , the user's accuracy for corn/soybean class increased to $83.8 \%$, but the producer's accuracy decreased to $54.3 \%$, the less balanced user's and producer's accuracies led to a lower kappa value (0.33). A higher threshold value of 0.7 led to a high user's accuracy (86.9\%) and a much lower producer's accuracy (34.0\%), so about $66 \%$ of actual corn/soybean pixels in the CDL were 

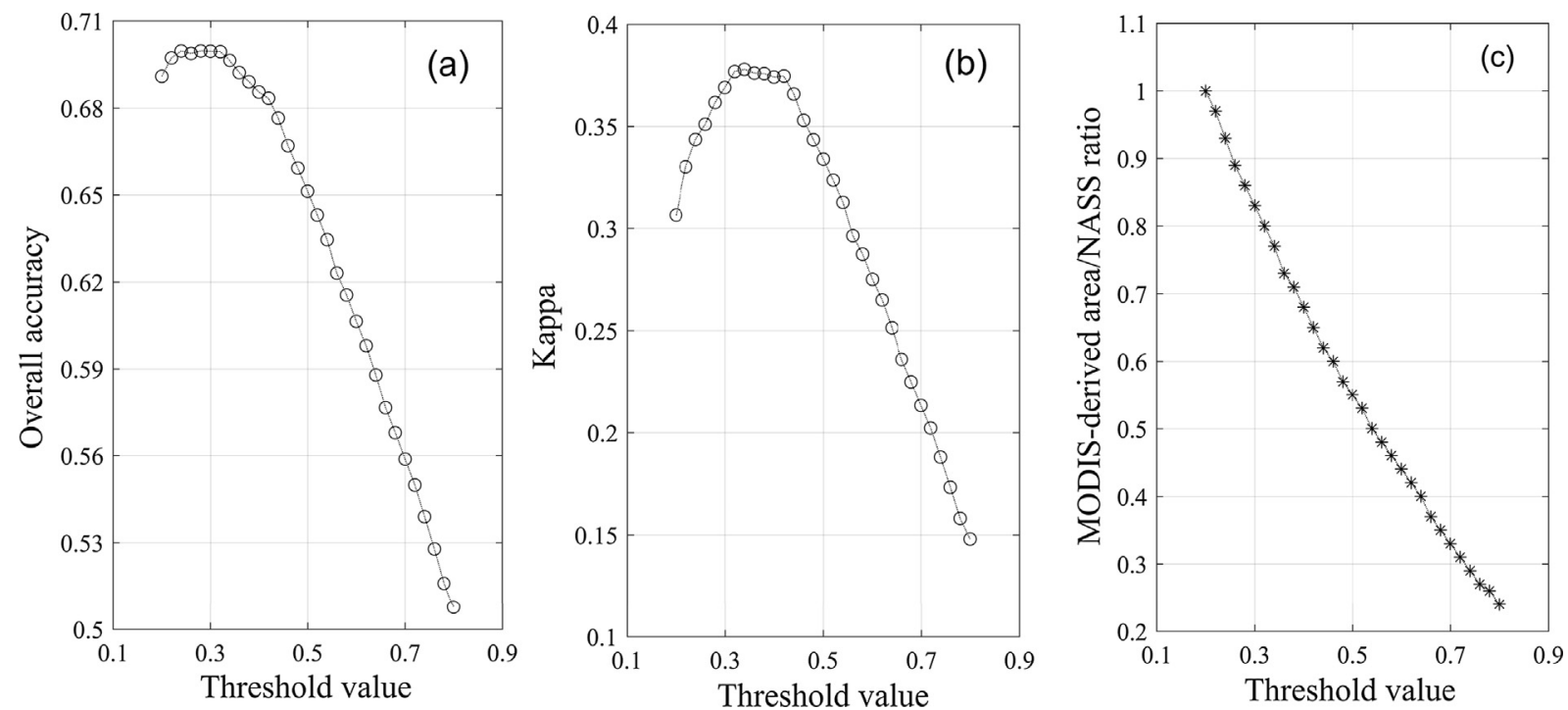

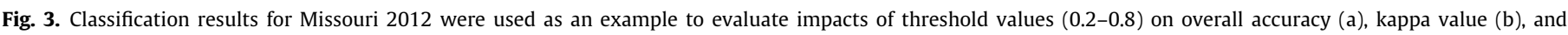
MODIS_area/NASS_area ratio (c).

Table 2

Comparison of confusion matrices of NN classification using three different threshold values of $0.3,0.5$, and 0.7 .

\begin{tabular}{|c|c|c|c|c|}
\hline \multirow[t]{2}{*}{ Threshold $=0.3$} & \multicolumn{3}{|l|}{ CDL reference } & \multirow[t]{2}{*}{$\%$ correct } \\
\hline & Corn/soybean & Other & Total & \\
\hline Corn/soybean & 37,148 & 11,051 & 48,199 & 77.1 \\
\hline Other & 12,385 & 18,304 & 30,689 & 59.6 \\
\hline Total & 49,533 & 29,355 & 78,888 & \\
\hline \%correct & 75.0 & 62.4 & Overall $=70.2 \%$ & Карpa $=0.37$ \\
\hline Threshold $=0.5$ & Corn/soybean & Other & Total & $\%$ correct \\
\hline Corn/soybean & 26,906 & 5194 & 32,100 & 83.8 \\
\hline Other & 22,627 & 24,161 & 46,788 & 51.6 \\
\hline Total & 49,533 & 29,355 & 78,888 & \\
\hline \%correct & 54.3 & 82.3 & Overall = 64.7\% & Карра $=0.33$ \\
\hline Threshold $=0.7$ & Corn/soybean & Other & Total & $\%$ correct \\
\hline Corn/soybean & 16,835 & 2544 & 19,379 & 86.9 \\
\hline Other & 32,698 & 26,811 & 59,509 & 45.1 \\
\hline Total & 49,533 & 29,355 & 78,888 & \\
\hline \%correct & 34.0 & 91.3 & Overall = 55.3\% & Карра $=0.21$ \\
\hline
\end{tabular}

Table 3

Ratios between the CDL-derived corn/soybean crop acreage and the NASS acreage statistics for 2010-2015.

\begin{tabular}{|c|c|c|c|c|}
\hline & Kansas & Minnesota & Missouri & South Dakota \\
\hline 2010 & 0.80 & 1.00 & 0.87 & 1.01 \\
\hline 2011 & 0.80 & 1.04 & 0.82 & 1.06 \\
\hline 2012 & 0.76 & 1.01 & 0.85 & 1.01 \\
\hline 2013 & 0.81 & 1.04 & 0.85 & 1.01 \\
\hline 2014 & 0.73 & 1.05 & 0.85 & 1.02 \\
\hline 2015 & 0.76 & 1.01 & 0.84 & 1.00 \\
\hline Average & 0.78 & 1.02 & 0.85 & 1.02 \\
\hline
\end{tabular}

mis-classified as other lands. Z-statistic testing suggests these three classification results were significantly different from each other $(p<0.05)$.

\subsection{Integration of NASS statistics}

Table 3 shows the ratio between the CDL-derived corn/soybean crop acreage (based on the $250 \mathrm{~m}$ MODIS grid) and the NASS statistics for four states for the years 2010-2015. For Missouri, the average ratio was around 0.85 which matched well with the previously identified optimal threshold value for image classification performance (Fig. 3c). The average ratio for Kansas was 0.78 . Such ratio values suggest that remote sensing pixel counting could lead to downward biased estimation for some states. However, for Minnesota and South Dakota, the ratios were slightly above 1. More importantly, for 2010-2015, we observed relatively consis- 

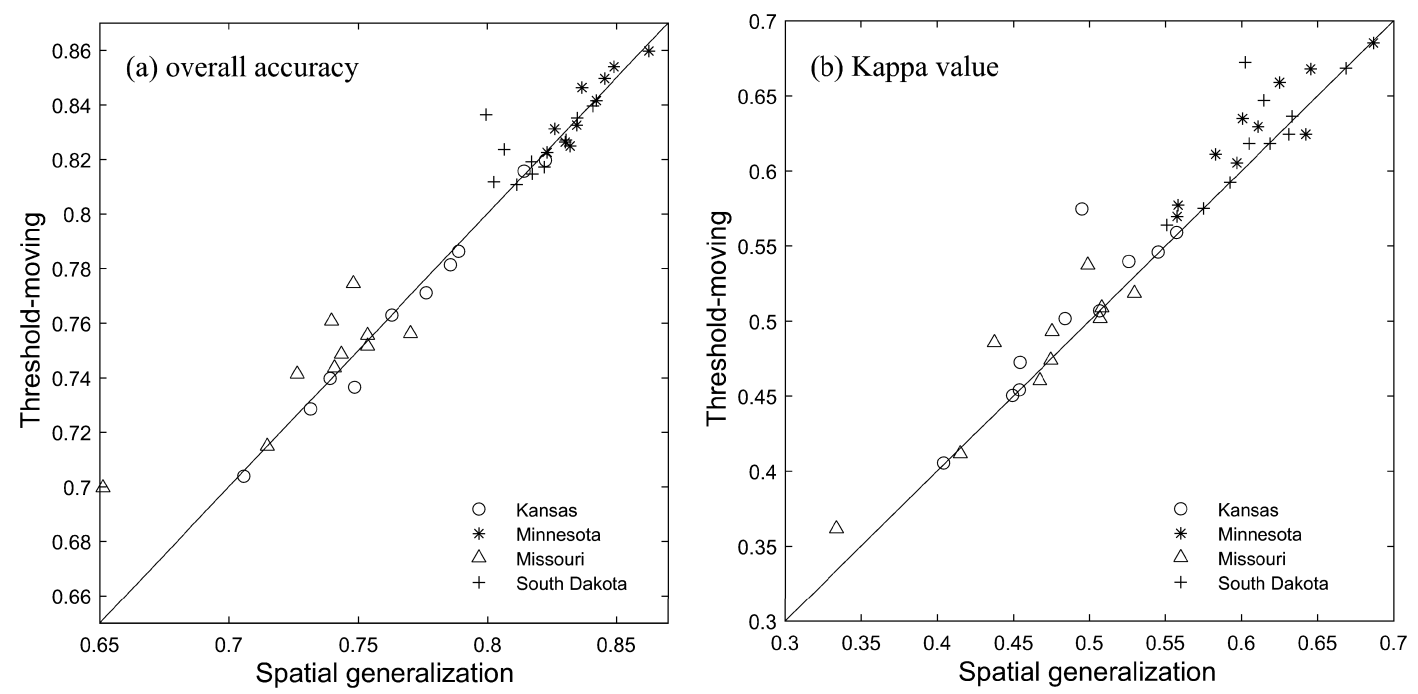

Fig. 4. Comparison of overall accuracy (a) and kappa coefficients (b) using simple NN spatial generalization (threshold $=0.5$ ) and the threshold-moving approach.

tent ratio values for Minnesota, Missouri, and South Dakota. Such results suggest that it might be feasible to use the average ratio and the annual state-level NASS statistics to estimate total number of corn/soybean pixels for each year - a potential solution to automatically select a threshold value in the NN classification.

We applied the automated NN threshold selection method to four states and compared classification accuracy statistics to those derived from the spatial generalization approach which always uses 0.5 as a threshold (Fig. 4). For all study years from 2006 to 2015, the average overall accuracy was 0.789 and 0.793 for spatial generalization and threshold-moving approach, respectively. For most study years and states, the performance from the two approaches were quite similar. The main improvement can be seen for Missouri and South Dakota, where the threshold-moving approach improved as much as $4 \%$ of overall accuracy. The average kappa value was 0.54 and 0.56 for the spatial generalization and the threshold-moving approach, respectively. Above $70 \%$ of testing states-years showed improved classification accuracy using the threshold-moving method. The largest improvement of kappa value was approximately 0.08 . A closer look at the confusion matrixes showed that higher kappa coefficients were often linked to lower and more balanced commission-omission errors for corn/soybean and the other crop class.

\subsection{Corn/soybean planting intensity from 2001 to 2015}

Comparisons of classification performance for the years 20062015 suggested that the threshold-moving approach outperformed the spatial generalization method. We thus used the thresholdmoving method and the annual NASS corn/soybean acreage statistics to generate annual corn/soybean map products for 2001-2015. Although complete CDL products were available for 2006-2015, we still generated the $250 \mathrm{~m}$ MODIS-based corn/soybean maps to maintain the data consistency. The $250 \mathrm{~m}$ corn/soybean maps were further aggregated to $5 \mathrm{~km}$ resolution to compute crop intensities to support the 15-year trend analysis. To further verify that MODIS-derived crop intensities approximate the CDL-derived crop intensities, we conducted a correlation analysis to compare MODIS- and CDL-derived corn/soybean proportions at the $5 \mathrm{~km}$ resolution for North Dakota and Iowa, where the CDLs are available for all study years from 2001 to 2015. The $r$ value was 0.97, suggesting excellent agreement between the two crop intensity datasets.
Fig. 5 depicts window-by-window slope coefficients of Sen's slope estimation developed for 2001-2015. Note that only windows that showed significant $(p<0.05)$ upward/downward trends based on the Mann-Kendall test were included for trend model development. The highest slope coefficient was 0.068 . For a 15year time period, the average increase rate of $6.8 \%$ per year was considerably high. A total of $61105 \times 5 \mathrm{~km}$ windows (around $152,750 \mathrm{~km}^{2}$ ) showed at least $1 \%$ of average annual increase in corn/soybean intensity. Areas showing fast increases of corn/soybean intensities were mainly located in North Dakota, South Dakota, and the western portion of Minnesota. We conducted additional correlation analysis to compare MODIS-derived change rates (or slope coefficients) with CDL-derived rates for North Dakota and Iowa for the 2001 to 2015 study period, and the $r$ value was 0.91 . Such high correlation indicates that MODIS-derived change rates were consistent with CDL-derived rates. Furthermore, the average change rate derived for the entire study area appeared to be similar to results reported by Wright and Wimberly (2013).

\section{Discussion}

Performance of NN spatial generalization using multi-temporal MODIS data is dependent on several interrelated factors: classification scheme, spectral-temporal characteristics of land cover classes in both training and testing regions, NN configuration and parameter selection, training method (e.g., cross-validation), and threshold value used to label target classes. In this study, a simple twoclass classification scheme was used following Wright and Wimberly's (2013) cropland change research for the WCB. The combined corn/soybean class provides a meaningful thematic class to characterize major cropland change patterns in the WCB and it also potentially improves classification accuracy by removing confusion between corn and soybean classes. Low classification accuracy may confound the long-term cropland change trends. The trained NN classifiers for North Dakota, Nebraska, and Iowa were directly applicable to corn/soybean mapping for Minnesota and South Dakota without integrating the NASS statistics and threshold-moving. Such results suggest that corn/soybean's spectral-temporal signals from these states may have similar characteristics.

We noticed that overall accuracies were substantially lower for Missouri (overall accuracy $>0.65$ ) and Kansas (overall accuracy $>0.71$ ) compared to those of South Dakota and Minnesota 


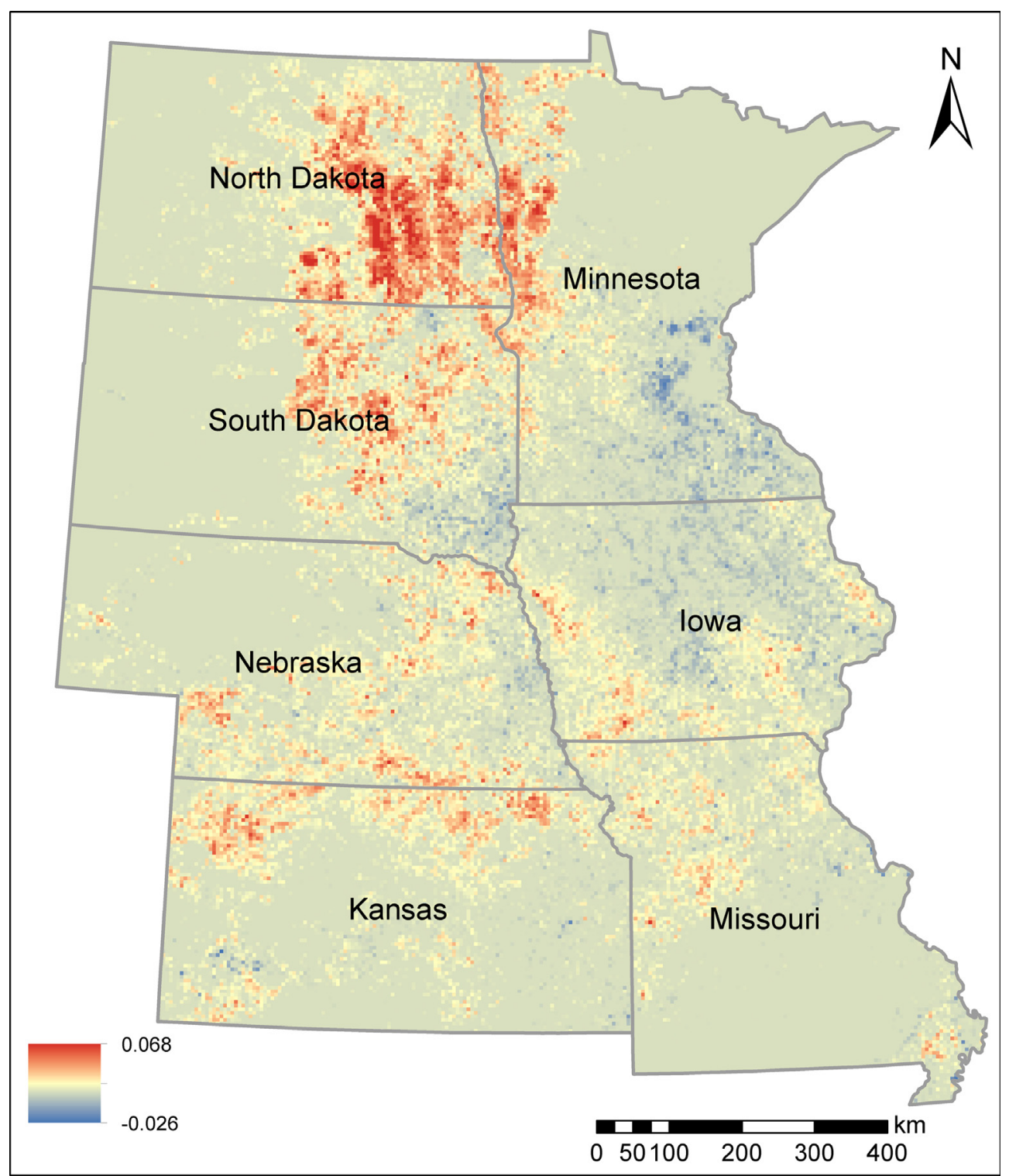

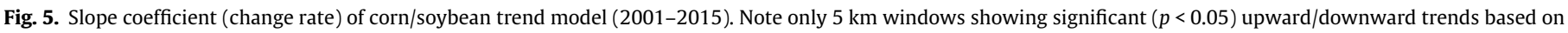
the Mann-Kendall test were used for trend model development.

(i.e., >80\% for all testing years from 2006 to 2015). For Missouri, using a default threshold value of 0.5 , remote sensing-estimated corn/soybean acreage was highly inconsistent with the NASS acreage statistics across different study years (i.e., ratio ranged from 0.43 to 0.83 for 2006-2015), which further proved the difficulty in spatial generalization of the NN classifier for corn/soybean mapping. In the southern portion of the WCB region (Missouri and Kansas), the warmer climate allows earlier planting of corn/soybean compared the northern states in the WCB (Ren et al., submitted for publication). The differences in crop planting dates may contribute to the poor performance of NN spatial generalization, especially since no training data came from areas in the south. Additionally, warmer climate in the southern portion of the WCB may allow more double-cropping (e.g., wheat-soybean) and a wider variety of summer crops, both of which will contribute to difficulty in NN spatial generalization. All these factors may contribute to large variations in crop phenology and lead to significant challenges in designing and implementing image classification algorithms. Previous studies have used ecoregion-based stratification of study areas to reduce phenological differences before image classification (e.g., Shao et al., 2010), however, such an approach typically requires training data points from each ecoregion and multiple classifiers need to be trained and validated. Additional types of neural networks (e.g., a radial basis functions or RBFs) and other advanced machine learning algorithms (e.g., Random Forest and Support Vector Machines) showed strong generalization performance for various remote sensing classification problems (Mountrakis et al., 2011; H. Wang et al., 2014, Q. Wang et al., 2014). For a future study, their spatial generalization effectiveness needs to be examined and compared with the NN classifier used in this study.

One important finding from our study is that threshold-moving, when combined with the NASS statistics, can improve corn/soybean mapping accuracy for certain study years (and states). The NN fuzzy output signals can be treated as the probability of the corn/soybean class, given the multi-temporal MODIS profiles. Users can select any threshold/probability value to label the corn/soybean class. This procedure clearly affects overall accuracy and involves a trade-off between commission error and omission error. A high threshold value (e.g., 0.8 ) may lead to very low commission 
error and high omission error. Depending on different applications, researchers may choose certain threshold values to maximize overall accuracy, kappa value, user's/producer's accuracy, or find optimal cut off points on the receiver operating characteristic (ROC) curve (Freeman and Moisen, 2008). None of these automated threshold selection methods can be directly applied to our spatial generalization tasks. For example, a default 0.5 threshold value generally led to the highest overall accuracy (kappa value) for the training region (North Dakota, Nebraska, and Iowa). However, the optimal threshold value for the testing region could be very different (e.g., 0.3 for Missouri, 2012). In this study, the NASS crop acreage statistics was used to determine the automated threshold selection. This method is particularly useful for generating MODISbased cropland maps backward in time since the NASS acreage statistics are readily available. The capability of near real-time mapping is less important in our study because our main purpose was to develop high quality long-term corn/soybean map products to support cropland change analysis.

The performance of the NN classifier is also dependent on the quality of training data. The CDLs were used as reference to extract training data points. CDL accuracies are high ( $>85 \%$ for most states in the study region) for corn and soybean classes. The accuracy for a combined corn/soybean class could be higher; thus CDL-derived training data points are sufficiently accurate for MODIS-based corn/soybean mapping. We note that MODIS EVI (Enhanced Vegetation Index) was also considered due to its lower signal saturation (Huete et al., 2002). However, we found some EVI data quality problem for year 2012 for our study region. Previous studies also suggested similar image classification performance using multitemporal NDVI or EVI (Shao and Lunetta, 2011; Wardlow and Egbert, 2010). Therefore, we focused on MODIS NDVI for our corn/soybean mapping efforts. In a recent study, Wang et al. (2012) indicated that sensor degradation of MODIS Terra led to slight but observable decline of NDVI during 2002 to 2010. Such a declining trend in NDVI may add uncertainty in multi-temporal image classification and subsequent change analysis. We expect that Terra MODIS calibration and reprocessing (Collection 6) will address this sensor degradation issue (Wang et al., 2012). To achieve high spatial generalization capability, a variety of NN configurations (e.g., number of nodes in the hidden layer) and other training protocols (learning rate, momentum) need to be examined. All of these settings can be examined under a cross-validation framework to find the optimal combination, thus they are not major concerns for our study. With improved MODIS signal calibration and advanced image classification algorithms, we think there is potential in temporal generalization in addition to our current efforts in spatial generalization. Specifically, further research should test whether a trained classifier for one specific year can be directly applied to another year to derived good classification performance. In a recent study, Zhong et al. (2016) developed an automated corn and soybean mapping method using crop phenology. Their approach highlights the use of decision rules based on expert inputs and differences in corn and soybean phenology. Such expert system has potential to be generalized across time. However, overall we think that a temporal generalization is a more challenging topic compared to a spatial generalization problem.

Our research indicates that the WCB region has experienced a high level of cropland intensification from 2001 to 2015. Annual increase of corn/soybean intensity reached as high as $6.8 \%$ for some $5 \times 5 \mathrm{~km}$ windows, which is consistent with results from Wright and Wimberly (2013). One contribution of our study is that we examined all seven WCB states with complete corn/soybean map products from 2001 to 2015. Future research should be implemented to extend our annual corn/soybean mapping efforts to the entire Midwestern region to examine cropland change trends and assess their impacts on water yield/quality, biodiversity, and ecosystem services in general. For the current study, we focused on per-pixel image classification method to generate binary corn/soybean maps to support change trend analysis. An alternate method is to estimate proportional corn/soybean cover using sub-pixel mapping algorithms (e.g., Chang et al., 2007) to support change trend analysis. The proportional cover estimation can reduce the impacts of landscape heterogeneity and provide more accurate corn/soybean proportions, which is particularly important for study areas where agricultural fields are relatively small, though large fields are most common in the WCB.

\section{Conclusions}

Using multi-temporal MODIS-NDVI as inputs, we developed annual corn/soybean map products through NN training and generalization. The existing high quality CDLs from a sub-region of the WCB were used as reference to extract training data points for NN training. The trained networks were directly applied to the other sub-regions to evaluate their spatial generalization effectiveness. With a default threshold value of 0.5 , we observed high variation of classification performance, and overall accuracy ranged from $0.65(\mathrm{kappa}=0.33)$ to $0.86(\mathrm{kappa}=0.69)$ for four states in the testing region, during the period 2006-2015. A thresholdmoving method was developed to improve NN spatial generalization performance. The adjustment of threshold values (e.g., 0.20.8 ) on the NN output signals affects the total number of MODIS pixels labeled as corn/soybean. When we integrated state-level NASS acreage statistics to guide the threshold selection, over 70\% of testing state-years showed improved kappa coefficients. The largest improvement of kappa value was approximately 0.08 . This threshold-moving method was applied to all study years from 2001 to 2015 to support long-term cropland change analysis. A total of $61105-\mathrm{km}$ windows showed significant $(p<0.05)$ increase of corn/soybean intensity, and the highest annual increase rate was $6.8 \%$.

\section{Acknowledgements}

The authors thank Norwegian Research Council for supporting our research. Funding was partially provided by Norwegian Research Council grant number 194051.

\section{References}

Atzberger, C., Eilers, P.H.C., 2011. Evaluating the effectiveness of smoothing algorithms in the absence of ground reference measurements. Int. J. Remote Sens. 32, 3689-3709.

Boryan, C., Yang, Z., Mueller, R., Craig, M., 2011. Monitoring US agriculture: the US department of agriculture, national agricultural statistics service, cropland data layer program. Geocarto Int. 26, 341-358.

Chang, J., Hansen, M.C., Pittman, K., Carroll, M., DiMiceli, C., 2007. Corn and soybean mapping in the United States using MODIS time-series data sets. Agron. J. 99 1654-1664.

Chen, Y., Song, X., Wang, S., Huang, J., Mansaray, L.R., 2016. Impacts of spatial heterogeneity on crop area mapping in Canada using MODIS data. ISPRS J. Photogramm. Remote Sens. 119, 451-461.

Congalton, R.G., 1991. Remote sensing and geographic information system data integration: error sources and. Photogramm. Eng. Remote Sens. 57, 677-687.

Doraiswamy, P.C., Stern, A.J., Akhmedov, B., 2007. Crop classification in the US Corn Belt using MODIS imagery. In: 2007 IEEE International Geoscience and Remote Sensing Symposium. IEEE, pp. 809-812.

Eilers, P.H.C., 2003. A perfect smoother. Anal. Chem. 75, 3631-3636.

Freeman, E.A., Moisen, G.G., 2008. A comparison of the performance of threshold criteria for binary classification in terms of predicted prevalence and kappa. Ecol. Modell. 217, 48-58.

Gallego, F.J., 2004. Remote sensing and land cover area estimation. Int. J. Remote Sens. 25, 3019-3047.

Hamed, K.H., 2008. Trend detection in hydrologic data: the Mann-Kendall trend test under the scaling hypothesis. J. Hydrol. 349, 350-363.

Hansen, M.C., Loveland, T.R., 2012. A review of large area monitoring of land cover change using Landsat data. Remote Sens. Environ. 122, 66-74. 
Huete, A., Didan, K., Miura, T., Rodriguez, E.P., Gao, X., Ferreira, L.G., 2002. Overview of the radiometric and biophysical performance of the MODIS vegetation indices. Remote Sens. Environ. 83, 195-213.

Johnson, D.M., 2010. The 2007 Cropland Data Layer.

Johnson, D.M., 2013. A 2010 map estimate of annually tilled cropland within the conterminous United States. Agric. Syst. 114, 95-105.

Johnson, D.M., Mueller, R., 2010. The 2009 cropland data layer. Photogramm. Eng. Remote Sens. 76, 1202-1205.

Kline, K.L., Singh, N., Dale, V.H., 2013. Cultivated hay and fallow/idle cropland confound analysis of grassland conversion in the Western Corn Belt. Proc. Natl. Acad. Sci. 110. E2863-E2863.

Lark, T.J., Salmon, J.M., Gibbs, H.K., 2015. Cropland expansion outpaces agricultural and biofuel policies in the United States. Environ. Res. Lett. 10, 44003.

Lunetta, R.S., Shao, Y., Ediriwickrema, J., Lyon, J.G., 2010. Monitoring agricultural cropping patterns across the Laurentian Great Lakes Basin using MODIS-NDVI data. Int. J. Appl. Earth Obs. Geoinf. 12, 81-88.

Moody, A., Gopal, S., Strahler, A.H., 1996. Artificial neural network response to mixed pixels in coarse-resolution satellite data. Remote Sens. Environ. 58, 329 343.

Mountrakis, G., Im, J., Ogole, C., 2011. Support vector machines in remote sensing: a review. ISPRS J. Photogramm. Remote Sens. 66, 247-259.

Provost, F., 2000. Machine learning from imbalanced data sets 101. In: Proceedings of the AAAI'2000 Workshop on Imbalanced Data Sets, pp. 1-3.

Ren, J., Campbell, J.B., Shao, Y., 2016. Spatial and temporal dimensions of agricultural land use changes, 2001-2012, East-Central Iowa. Agric. Syst. 148, 149-158.

Ren, J., Campbell, J.B., Shao, Y., 2016. Estimation of SOS and EOS for Midwestern US Corn and Soybean Crops. Manuscript submitted for publication.

Richard, M.D., Lippmann, R.P., 1991. Neural network classifiers estimate Bayesian a posteriori probabilities. Neural Comput. 3, 461-483.

Sahajpal, R., Zhang, X., Izaurralde, R.C., Gelfand, I., Hurtt, G.C., 2014. Identifying representative crop rotation patterns and grassland loss in the US Western Corn Belt. Comput. Electron. Agric. 108, 173-182.

Shao, Y., Campbell, J.B., Taff, G.N., Zheng, B., 2015. An analysis of cropland mask choice and ancillary data for annual corn yield forecasting using MODIS data. Int. J. Appl. Earth Obs. Geoinf. 38, 78-87.

Shao, Y., Lunetta, R.S., 2011. Sub-pixel mapping of tree canopy, impervious surfaces, and cropland in the Laurentian Great Lakes Basin using MODIS time-series data. Sel. Top. Appl. Earth Obs. Remote Sensing, IEEE J. 4, 336-347.
Shao, Y., Lunetta, R.S., 2012. Comparison of support vector machine, neural network, and CART algorithms for the land-cover classification using limited training data points. ISPRS J. Photogramm. Remote Sens. 70, 78-87.

Shao, Y., Lunetta, R.S., Ediriwickrema, J., Iiames, J., 2010. Mapping cropland and major crop types across the Great Lakes Basin using MODIS-NDVI data. Photogramm. Eng. Remote Sens. 76, 73-84.

Shao, Y., Lunetta, R.S., Wheeler, B., Iiames, J.S., Campbell, J.B., 2016. An evaluation of time-series smoothing algorithms for land-cover classifications using MODISNDVI multi-temporal data. Remote Sens. Environ. 174, 258-265.

Shao, Y., Taff, G.N., Walsh, S.J., 2011. Comparison of early stopping criteria for neural-network-based subpixel classification. IEEE Geosci. Remote Sens. Lett. 8, $113-117$.

Wang, D., Morton, D., Masek, J., Wu, A., Nagol, J., Xiong, X., Levy, R., Vermote, E., Wolfe, R., 2012. Impact of sensor degradation on the MODIS NDVI time series, Remote Sens. Environ. 119, 55-61.

Wang, H., Shao, Y., Kennedy, L.M., 2014. Temporal generalization of sub-pixel vegetation mapping with multiple machine learning and atmospheric correction algorithms. Int. J. Remote Sens. 35, 7118-7135.

Wang, O. Shi, W., Atkinson, P.M., 2014. Sub-pixel mapping of remote sensing images based on radial basis function interpolation. ISPRS J. Photogramm. Remote Sens. 92, 1-15.

Wardlow, B.D., Egbert, S.L., 2008. Large-area crop mapping using time-series MODIS 250 m NDVI data: an assessment for the US Central Great Plains. Remote Sens. Environ. 112, 1096-1116.

Wardlow, B.D., Egbert, S.L., 2010. A comparison of MODIS 250-m EVI and NDVI data for crop mapping: a case study for southwest Kansas. Int. J. Remote Sens. 31, 805-830.

Wardlow, B.D., Egbert, S.L., Kastens, J.H., 2007. Analysis of time-series MODIS 250 m vegetation index data for crop classification in the US Central Great Plains. Remote Sens. Environ. 108, 290-310.

Wright, C.K., Wimberly, M.C., 2013. Recent land use change in the Western Corn Belt threatens grasslands and wetlands. Proc. Natl. Acad. Sci. 110, 4134-4139.

Zhang J. Feng, L. Yao, F. 2014. Improved maize cultivated area estimation over a large scale combining MODIS-EVI time series data and crop phenological information. ISPRS J. Photogramm. Remote Sens. 94, 102-113.

Zhou, Z.-H., Liu, X.-Y., 2006. Training cost-sensitive neural networks with methods addressing the class imbalance problem. IEEE Trans. Knowl. Data Eng. 18, 63-77.

Zhong, L., Hu, L., Yu, L., Gong, P., Biging, G.S., 2016. Automated mapping of soybean and corn using phenology. ISPRS J. Photogramm. Remote Sens. 119, 151-164. 